\title{
Avaliação de um programa de traçado cefalométrico*
}

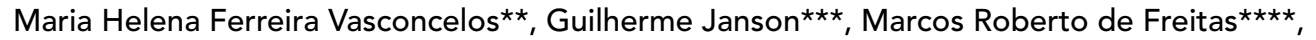

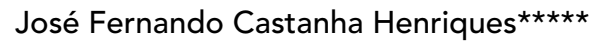

\section{Resumo}

Objetivo: avaliar o programa de traçado cefalométrico Radiocef 2.0 quanto à confiabilidade e precisão, comparativamente ao método manual e ao programa Dentofacial Planner 7.02 (Gold Standard). Metodologia: a amostra constou de 50 telerradiografias laterais de boa qualidade, de pacientes de ambos os gêneros, na faixa etária dos 11 aos 24 anos, pertencentes aos arquivos da disciplina de Ortodontia da Faculdade de Odontologia de Bauru, da Universidade de São Paulo. A partir das diferentes formas de medições executadas, 4 grupos experimentais foram obtidos: Grupo 1 (método manual); Grupo 2 (a partir da digitalização dos traçados no programa Radiocef 2.0); Grupo 3 (a partir da digitalização das radiografias no programa Radiocef 2.0); e Grupo 4 (a partir da digitalização dos pontos cefalométricos no programa Dentofacial Planner 7.02). Na composição do estudo, foram selecionadas medidas angulares e lineares. A comparação inter-grupos foi realizada por meio da análise de variância e do teste de Kruskal-Wallis. Resultados e conclusões: os programas mostraram-se comparáveis, sem diferenças estatisticamente significantes, ao nível de $5 \%$, possibilitando as seguintes conclusões: 1) o programa de traçado cefalométrico computadorizado Radiocef 2.0 pode ser confiavelmente utilizado como recurso auxiliar no diagnóstico, plano de tratamento, acompanhamento e avaliação de tratamentos ortodônticos, nos âmbitos clínico e/ou de pesquisa; e 2) este programa também pode ser confiavelmente utilizado para efetuar medições a partir da digitalização dos traçados, além da forma proposta pelo fabricante.

Palavras-chave: Cefalometria computadorizada. Traçado cefalométrico computadorizado. Programa de traçado cefalométrico.

\section{INTRODUÇÃO E REVISÃO DA LITERATURA}

No ano de 1931, a Ortodontia consagrou a era da cefalometria radiográfica, a partir dos históricos trabalhos de Broadbent ${ }^{14}$ e Hofrath ${ }^{32}$, apresentando ao mundo uma nova técnica radiográfica, utilizando o cefalostato. A telerradiogra- fia cefalométrica tornara-se então imprescindível ao diagnóstico, planejamento e avaliação dos resultados dos casos tratados ortodonticamente. Sua aplicação estendeu-se à área da pesquisa ortodôntica, onde são importantes a quantificação dos dados de pacientes de uma amostra,

* Artigo baseado na tese de doutorado da Cirurgiã-Dentista Maria Helena Ferreira Vasconcelos apresentada à Faculdade de Odontologia de Bauru, USP.

** Especialista em Radiologia e Ortodontia, Mestre em Patologia Bucal, Doutora em Ortodontia pela Faculdade de Odontologia de Bauru - USP.

** Professor Associado da Disciplina de Ortodontia da USP-Bauru, Coordenador do Curso de Mestrado em Ortodontia da USP-Bauru e Member of the Royal College of Dentists of Canada (MRCDC).

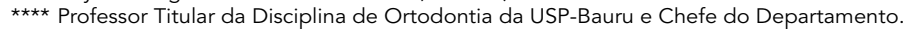

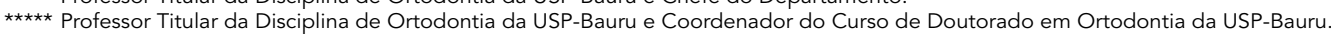


assim como a padronização da técnica radiográfica. Muitas análises cefalométricas foram pioneiramente propostas por diferentes autores, visando conhecer os padrões da morfologia craniofacial, estimar os métodos de tratamento e quantificar os resultados obtidos ${ }^{59,70,71,74}$.

Alguns autores preocuparam-se em avaliar a correção de erros nas radiografias cefalométricas, enumerando os fatores capazes de produzir distorções em suas imagens ${ }^{1,26}$. Outros preocuparam-se em avaliar e adequar a utilização dos conceitos de normalidade das medidas cefalométricas, assim como suas limitações, quando da realização do diagnóstico e do plano de tratamento ${ }^{10,29,31,43,65}$. Yen ${ }^{77}$, em 1960, preocupou-se no ensinamento minucioso da anatomia radiográfica e na demarcação dos pontos anatômicos nas radiografias cefalométricas em norma lateral e frontal.

Em 1981, Ricketts ${ }^{62}$ publicou uma revisão sobre a história da cefalometria, distinguindo cinco etapas históricas: 1) padronização da técnica radiográfica; 2) aplicação clínica; estabelecimento de pontos e planos de referência; 3) descrição morfológica e tipificação dos casos; 4) previsão do crescimento e plano de tratamento; e 5) confusão entre pontos para descrição de reparos anatômicos e pontos para análise de crescimento. Após os primeiros cinqüenta anos de aplicação deste recurso, o autor discorreu a respeito das perspectivas da aplicação clínica da cefalometria, inclusive em terceira dimensão e análise computadorizada, as quais haviam sido há pouco tempo introduzidas.

Durante muito tempo, o método manual foi o único utilizado para a execução do traçado cefalométrico e obtenção das medições angulares e lineares necessárias em sua interpretação. A principal desvantagem deste método reside no fato de demandar um tempo relativamente longo, na maioria das vezes, do próprio ortodontista.

Os constantes avanços tecnológicos na área da computação, aliados aos avanços científicos da radiologia odontológica, resultaram no desenvolvimento de programas computadorizados, destinados a efetuar os traçados e as medições cefalométricas, além da execução de diferentes tipos de análises. Com isto, no final da década de 60 e início dos anos 70, a cefalometria radiográfica começou a assumir um outro aspecto, pois o computador tornara-se mais um aliado na busca de informações quantitativas, concernentes ao diagnóstico ortodôntico e aos eventos de crescimento e desenvolvimento craniofacial.

Os pesquisadores australianos Barrett, Brown e $\mathrm{McNulty}^{4}$ descreveram um sistema computadorizado para medições dentárias e craniofaciais, explicando que a essência do método encontra-se na localização dos pontos em relação aos eixos de coordenadas. No ano seguinte Ricketts ${ }^{60}$ reconheceu esta fase como sendo a da comunicação cibercultural, exprimindo que o computador tornara-se essencial à cefalometria radiográfica devido à grande quantidade de informação acrescentada aos cefalogramas, e que a pesquisa científica havia tomado uma injeção de catalisador ${ }^{61}$. Krogman ${ }^{44}$ relatou a opinião dos participantes de um simpósio, promovido pela American Association of Orthodontics sobre o assunto: o futuro promissor da cefalometria computadorizada fundamentava-se no fato desta cumprir dois requisitos básicos necessários à pesquisa; a quantificação e a padronização dos dados. Em 1973 foi lançado o programa RMDS (Rocky Mountain Data Systems), proposto por Ricketts ${ }^{63}$. Em 1976, Chebib, Cleall e Carpenter ${ }^{17}$, da Universidade de Illinois, divulgaram o programa Chephalometric Records Analysis Program. Faber, Burstone e Solonche ${ }^{23}$, em 1978, mencionaram que a utilização do computador na ciência odontológica era relativamente pequena, envolvendo duas áreas apenas; estudos do crescimento e desenvolvimento, e efetuando comparações entre as medidas dos pacientes e as medidas dos padrões faciais normais. Neste ano também, os pesquisadores do Instituto Karolinska, Bergin, Hallenberg e Malmgren ${ }^{9}$ apresentaram o método 
de análise cefalométrica computadorizada utilizado por eles. Em 1979, Houston ${ }^{33}$ descreveu os componentes do sistema por ele empregado, esclarecendo os três programas integrados do método. Baumrimd e Miller ${ }^{8}$ divulgaram, em 1980, o método chamado The University of California at San Francisco Combined Head Film Analysis. Em 1981, Bondevik, Rosler e Slagsvold ${ }^{12}$ apresentaram o sistema Cm-1, desenvolvido em conjunto com o Instituto norueguês de pesquisa industrial, para medições em radiografias e modelos. Konchak e Koehler ${ }^{42}$, pesquisadores canadenses, apresentaram em 1985 o programa denominado Ucsd Pascal. A partir deste ano os programas desenvolvidos passaram a empregar as imagens digitais, sendo que Jackson, Dickson e Birnie ${ }^{37}$ foram os primeiros a discutirem os princípios de captura, armazenamento e processamento da imagem na radiologia digital, bem como sua aplicação em cefalometria. Em 1989, por meio do trabalho de Hing ${ }^{30}$, teve-se o conhecimento do lançamento do programa Quick Ceph, atualmente comercializado na versão 2000, lançada em 1999, durante o encontro da American Association of Orthodontists, em San Diego. Em 1990, Walker ${ }^{75}$ publicou um artigo descrevendo o programa Dentofacial Planner, lançado no ano anterior, representando atualmente um dos programas mais utilizados para pesquisas em cefalometria. Em 1991, Oliver ${ }^{55}$ apresentou o programa Cardiff Cephalometrics, comparando-o ao Imscan System ${ }^{36}$, desenvolvido no Royal Berkshire Hospital. Halazonetis ${ }^{28}$, de Atenas, lançou o programa Viewbox, em 1994, em conjunto com a Microsoft Corporation. Rudolph, Sinclair e Coggins ${ }^{64}$, em 1998, apresentaram o método de identificação automática dos pontos cefalométricos, onde a localização dos pontos é feita através de um método de reconhecimento de padrões, utilizando-se da espectroscopia espacial.

No Brasil, em 1996, Toigo, Moro e Vigorito ${ }^{72}$ apresentaram um programa computadorizado exclusivamente idealizado para a análise de Petrovic/Lavergne. Atualmente, são comercializados al- guns programas de traçado cefalométrico, a maioria operando com imagens digitais. Os programas Orto Manager ${ }^{68}$, Radiocef ${ }^{57}$ e Cef- $\mathrm{X}^{15}$ representam os programas nacionais mais modernamente comercializados na atualidade. Dentre estes, o Orto Manager é comercializado em conjunto com um programa de gerenciamento de consultório ortodôntico. O Radiocef representa um dos mais utilizados, principalmente pelas clínicas radiológicas e de documentação ortodôntica; e o Cef-X foi recentemente lançado, durante o Congresso da Sociedade Paulista de Ortodontia, em outubro de 2003.

Como o método de análise cefalométrica computadorizada conquistou a preferência da maioria dos ortodontistas brasileiros e pesquisadores da área, tal como os norte-americanos e europeus, e os programas nacionais vêm sendo desenvolvidos e constantemente melhorados, percebeu-se a necessidade de avaliar sua precisão, selecionando-se para isto o programa Radiocef $2.0^{57}$, comparativamente ao método manual e a outro programa internacional, o Dentofacial Planner 7.0222, de precisão já comprovada na literatura, e utilização freqüente em pesquisas $3,5,21,24,39,40,41,48,51,56,76$.

\section{MATERIAL E MÉTODOS Material}

A amostra constou de 50 telerradiografias cefalométricas laterais, do arquivo da Disciplina de Ortodontia da Faculdade de Odontologia de Bauru, da Universidade de São Paulo. Os pacientes radiografados encontravam-se na faixa etária compreendida entre os 11 e 24 anos de idade, sendo 27 do gênero feminino e 23 do gênero masculino. Todas as radiografias apresentavam contraste e nitidez adequados e boa visualização dos ápices dos incisivos centrais superiores e inferiores, representando diferentes tipos de má oclusão e de crescimento facial.

As 50 telerradiografias foram medidas de quatro maneiras distintas, pela mesma pesquisadora calibrada, doutoranda em Ortodontia, caracterizando 
4 grupos experimentais: Grupo 1 - medições lineares e angulares nas 50 radiografias, pelo método manual; Grupo 2 - medições realizadas pelo programa Radiocef 2.0 (Radiomemory - Rua Timbiras, 1560, Belo Horizonte/MG, Brasil), a partir da digitalização dos traçados das 50 telerradiografias realizados manualmente; Grupo 3 - medições realizadas pelo programa Radiocef 2.0, a partir da digitalização das 50 telerradiografias; e Grupo 4 - medições realizadas pelo programa Dentofacial Planner 7.02 (Dentofacial Soft. Inc. - Simcoe Street, Toronto, Canadá), a partir da digitalização dos traçados das 50 telerradiografias realizados manualmente, sobre a mesa digitalizadora.

Para a obtenção dos traçados cefalométricos e posteriores medições em todos os grupos experimentais foram necessários os seguintes materiais e equipamentos: papel de acetato transparente Ultraphan, de $0,07 \mathrm{~mm}$ de espessura, sobre um negatoscópio, em uma sala obscurecida; fita adesiva transparente; lapiseira de grafite macia $0,5 \mathrm{~mm}$; borracha branca macia; régua de $20 \mathrm{~cm}$; esquadro; transferidor de $180^{\circ}$; microcomputador; impressora; mesa digitalizadora, modelo Accugrid A3OTL.F - Numonics Corp. (Numonics Corp. 101 Commerce Dnve. Montgomeryville, PA, E.U.A.); scanner com leitor de transparência, da marca comercial Microtek (Microtek International., Inc. $n^{\circ}$ 6, Industry. East Road 3. Science-based Industry Park. Hsinchu, Taiwan), modelo Scan Maker II SP, calibrado para escalas de cinza, resolução de 75 dpi e tamanho da imagem 100\%; programa para traçado cefalométrico computadorizado Radiocef 2.0, instalado no microcomputador, em associação com o scanner acima citado; programa para traçado computadorizado Dentofacial Planner 7.02, instalado no microcomputador, em associação com a mesa digitalizadora acima citada.

\section{Métodos}

As medidas selecionadas para o estudo totalizaram 27, em 5 categorias distintas, descritas a seguir: medidas angulares - SNA, SNB, ANB, 1.NA,
-. NB, $\overline{1} . \underline{1}$, PoOr.GoGN, SN.GoMe, N.S.Gn e H.NB; medidas de comprimento - Co-A, Co-Gn, ENA-Me, S-N, S-Ar, N-Me e S-Go; distâncias horizontais - A-Nperp e P-Nperp; distâncias verticais - N-ENA e ENA-Gn; distâncias ponto-linha 1-NA, $\overline{1}-\mathrm{NB}, \mathrm{P}-\mathrm{NB}$ e H-nariz; diferença - diferencial maxilo-mandibular (Co-Gn - Co-A) e proporção - S-Go/N-Me x 100.

\section{Obtenção das medidas cefalométricas}

\section{Medidas do Grupo G1}

No grupo Gl, os traçados e as medidas cefalométricas foram obtidas manual e diretamente no papel Ultraphan. Os desenhos das estruturas anatômicas e os pontos cefalométricos foram localizados utilizando-se o negatoscópio, em uma sala obscurecida. Um grupo de no máximo 10 radiografias foi trabalhado a cada dia, de maneira a evitar a fadiga do operador, minimizando a chance de erros casuais acontecerem. Os dados foram anotados em uma ficha e manualmente transportados para uma planilha de trabalho do programa Excel 97 (USA, http://www.microsoft.com), para posterior tratamento estatístico destes.

\section{Medidas do Grupo G2}

Para a obtenção das medidas do Grupo G2, os traçados realizados manualmente, e utilizados para as medições do Grupo 1, foram digitalizados por meio do scannere utilizados para as medições computadorizadas, pelo programa Radiocef 2.0. A partir destas imagens digitalizadas, realizou-se a marcação dos pontos com a ajuda do cursor do mouse, diretamente na tela do monitor. Finalizada esta etapa, os dados foram calculados e fornecidos pelo programa, em um modelo de ficha relatório, emitido e impresso para cada uma das radiografias. As medidas contidas nestes relatórios foram manualmente transferidas e armazenadas no programa Excel 97, em procedimento semelhante ao do Grupo Gl, para posterior tratamento estatístico destes dados.

Anteriormente às etapas das medições com- 
Tabela 1 - Média (X), desvio padrão (S) e nível de significância (P) das medidas obtidas nos grupos G1, G2, G3 e G4, pela análise de variância (ANOVA).

\begin{tabular}{|c|c|c|c|c|c|c|c|c|c|}
\hline \multirow{2}{*}{$\begin{array}{c}\text { Medidas } \\
\text { cefalométricas }\end{array}$} & \multicolumn{2}{|c|}{ G1 } & \multicolumn{2}{|c|}{ G2 } & \multicolumn{2}{|c|}{ G3 } & \multicolumn{2}{|c|}{ G4 } & \multirow{2}{*}{$\mathbf{P}$} \\
\hline & $\mathbf{X}$ & S & $\mathbf{X}$ & S & $\mathbf{x}$ & $\mathbf{S}$ & $\mathbf{X}$ & S & \\
\hline PoOr.GoGn (graus) & 24,60 & 4,71 & 24,50 & 4,71 & 24,20 & 4,94 & 24,60 & 4,76 & 0,9654 \\
\hline Co-Gn (mm) & 118,20 & 7,39 & 118,30 & 7,37 & 118,80 & 7,88 & 117,90 & 7,38 & 0,9437 \\
\hline S-Go (mm) & 77,30 & 7,38 & 77,00 & 7,24 & 79,30 & 7,74 & 77,10 & 7,35 & 0,3532 \\
\hline P-Nperp (mm) & $-3,91$ & 7,05 & $-4,49$ & 7,01 & $-5,72$ & 7,14 & $-4,48$ & 6,97 & 0,6235 \\
\hline$\overline{1}$-NB (mm) & 4,47 & 2,79 & 4,42 & 2,83 & 4,64 & 2,91 & 4,34 & 2,81 & 0,9606 \\
\hline Diferença (mm) & 26,70 & 5,10 & 26,40 & 4,63 & 25,90 & 4,75 & 26,50 & 4,77 & 0,8611 \\
\hline
\end{tabular}

Tabela 2 - Mediana (Md) e nível de significância (P) das medidas obtidas nos grupos G1, G2, G3 e G4, pelo teste de Kruskal-Wallis.

\begin{tabular}{|c|c|c|c|c|c|}
\hline $\begin{array}{c}\text { Medidas } \\
\text { cefalométricas }\end{array}$ & $\begin{array}{l}\text { G1 } \\
\text { Md }\end{array}$ & $\begin{array}{l}\text { G2 } \\
\text { Md }\end{array}$ & $\begin{array}{l}\text { G3 } \\
\text { Md }\end{array}$ & $\begin{array}{l}\text { G4 } \\
\text { Md }\end{array}$ & $\mathbf{P}$ \\
\hline SNA (graus) & 82,0 & 82,3 & 82,5 & 81,9 & 0,7617 \\
\hline SNB (graus) & 78,5 & 78,7 & 79,1 & 78,5 & 0,9580 \\
\hline ANB (graus) & 4,00 & 3,67 & 4,06 & 3,75 & 0,9281 \\
\hline 1.NA (graus) & 23,8 & 24,0 & 23,9 & 23,9 & 0,8919 \\
\hline$\overline{1}$. NB (graus) & 26,8 & 26,4 & 27,4 & 26,6 & 0,9383 \\
\hline$\overline{1} .1$ (graus) & 125,0 & 125,3 & 123,7 & 125,5 & 0,8819 \\
\hline $\begin{array}{c}\text { SN.GoMe } \\
\text { (graus) }\end{array}$ & 34,0 & 34,4 & 33,1 & 34,5 & 0,6467 \\
\hline N.S.Gn (graus) & 67,0 & 66,4 & 66,4 & 66,7 & 0,8509 \\
\hline H.NB (graus) & 13,5 & 13,3 & 13,3 & 13,2 & 0,9518 \\
\hline Co-A (mm) & 90,3 & 90,9 & 92,0 & 90,8 & 0,5831 \\
\hline Ena-Me (mm) & 68,0 & 67,6 & 67,7 & 67,6 & 0,9935 \\
\hline S-N (mm) & 72,8 & 72,7 & 71,9 & 72,4 & 0,8971 \\
\hline S-Ar (mm) & 35,0 & 34,8 & 36,3 & 34,8 & 0.3504 \\
\hline $\mathrm{N}-\mathrm{Me}$ (mm) & 120,0 & 119,2 & 119.2 & 119,5 & 0,9612 \\
\hline A-Nperp (mm) & 0,75 & 0,74 & 0,24 & 0,65 & 0,8728 \\
\hline N-Ena (mm) & 53,0 & 53,0 & 53,1 & 52,9 & 0,9993 \\
\hline Ena-Gn (mm) & 64,8 & 64,3 & 64,2 & 64,6 & 0,9975 \\
\hline 1-NA (mm) & 4,50 & 4,36 & 4,42 & 4,30 & 0,9855 \\
\hline P-NB (mm) & 1,50 & 1,45 & 1,53 & 1,65 & 0,9320 \\
\hline H-nariz $(\mathrm{mm})$ & 3,25 & 3,21 & 3,33 & 3,00 & 0,9655 \\
\hline Proporção (\%) & 64,6 & 64,4 & 65,7 & 64,6 & 0,2015 \\
\hline
\end{tabular}
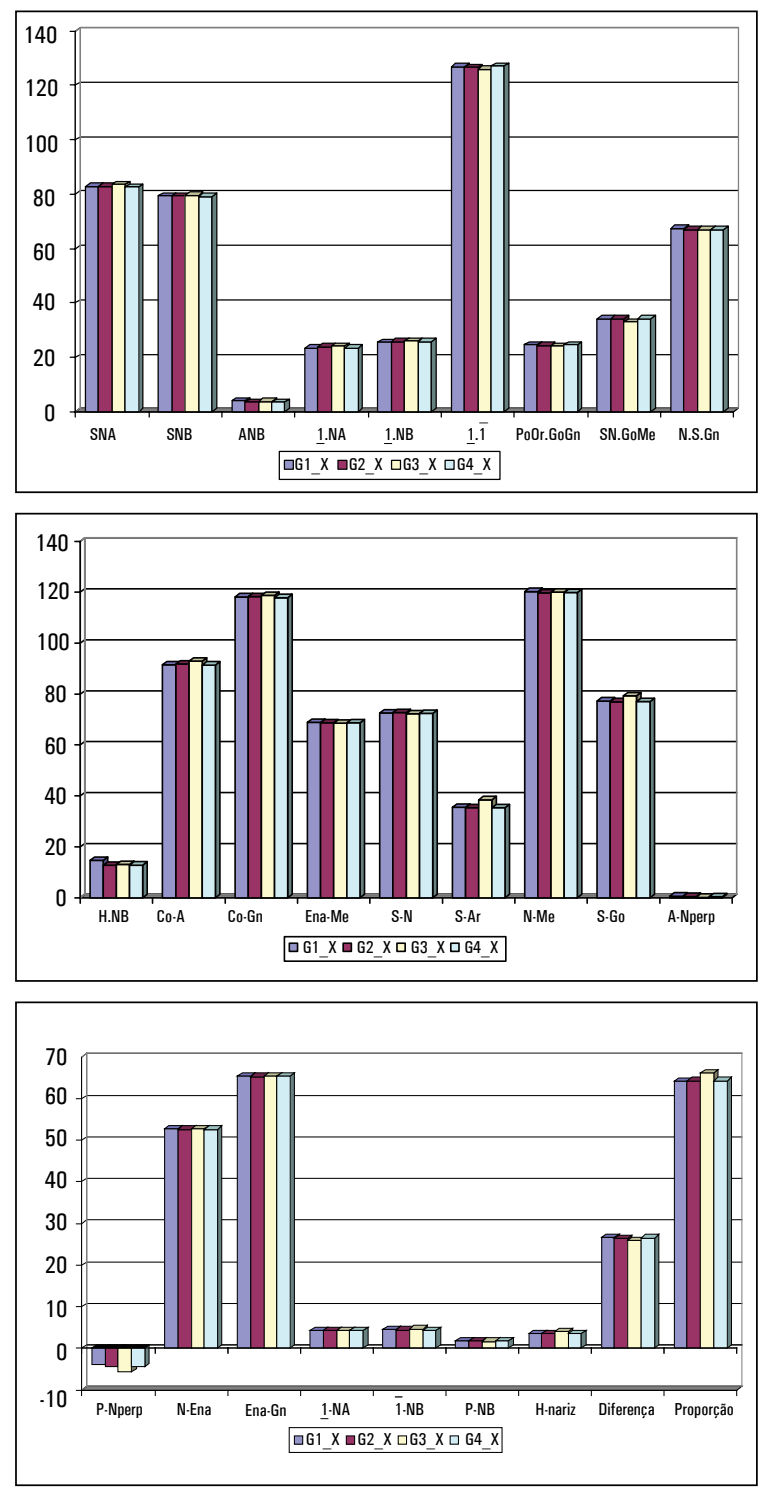

GRÁFICO 1, 2, 3 - Representação gráfica dos valores médios obtidos nos 4 grupos experimentais. 
putadorizadas, os programas Radiocef 2.0 e Dentofacial Planner 7.02 necessitaram ser ajustados, de modo a realizarem apenas as medições selecionadas neste estudo. Também cuidou-se para que ambos estivessem realizando as mesmas formas de medições.

\section{Medidas do Grupo G3}

À semelhança do Grupo G2, as medidas do Grupo G3 foram obtidas a partir de imagens digitalizadas, pelo scanner, das próprias telerradiografias, de acordo com o recomendado pelo fabricante. Os passos de marcação dos pontos, obtenção e arquivamento dos dados foram os mesmos descritos para o Grupo G2.

\section{Medidas do Grupo G4}

No grupo G4, as medidas foram efetuadas pelo programa Dentofacial Planner 7.02. Os traçados cefalométricos manuais, utilizados no Grupo G1, foram adaptados à mesa digitalizadora, onde se realizou a digitalização dos pontos, com o cursor próprio da mesa.

Assim como nos Grupos G2 e G3, o programa processou os dados, fornecendo os relatórios individuais das radiografias. Os dados destes relatórios foram exportados coletiva e diretamente para o programa Excel 97, recurso disponível neste programa.

Com todos os dados arquivados, realizaram-se os testes estatísticos entre os grupos.

\section{ANÁLISE ESTATÍSTICA}

$\mathrm{O}$ erro intra-examinador foi averiguado por meio de novos traçados e remedições de 10 radiografias da amostra, aleatoriamente escolhidas, da mesma maneira descrita anteriormente, para os 4 grupos experimentais, após 4 meses. Os dados obtidos nos tempos T1 e T2 foram comparados pelo teste $t$ pareado, com $\mathrm{p}<0,05$, representando a busca dos erros sistemáticos, no programa Sigma Stat $4.0^{38}$. Os erros casuais foram verificados utilizando-se a fórmula de Dahlberg ${ }^{19}$.
Os dados obtidos nos 4 grupos experimentais foram comparados pelo programa Sigma Stat, $4.0^{38}$, por meio da análise de variância a um critério (ANOVA), com $\mathrm{p}<0,05$. As medidas cujos valores não se enquadraram na curva de normalidade foram comparadas pelo teste de Kruskal-Wallis. Quando da observação de resultados significantes, previu-se a realização do teste de Tukey ou do teste de Dunn, para a localização exata destas possíveis ocorrências.

\section{RESULTADOS}

O resultado da pesquisa do erro intra-examinador acusou significância estatística em $15,7 \%$ das medidas efetuadas. No teste de Dahlberg, apenas 3 medidas acusaram valores acima de $1 \mathrm{~mm}$.

Os resultados da comparação entre os grupos experimentais encontram-se expostos nas tabelas 1 e 2 e nos gráficos 1,2 e 3.

\section{DISCUSSÃO}

A análise minuciosa dos resultados obtidos na comparação entre os 4 grupos experimentais permitiu observar valores muito próximos para as médias, medianas e desvios padrão, refletindo em valores de $\mathrm{P}$ não significantes para todas as medidas. A partir destes resultados, não foi necessária a realização dos testes de Tuckey ou de Dunn.

Com isto, concluiu-se que a forma de obtenção das medidas cefalométricas selecionadas não diferiu conforme o método escolhido, podendo-se afirmar que todos estes métodos são comparáveis. Estes resultados concordaram com os de outros trabalhos, comparando métodos e programas de traçado cefalométrico distintos ${ }^{5,16,34,37,50,55,66}$, tendo discordado apenas dos trabalhos de Forsyth et al. ${ }^{25}$, onde as medidas obtidas em radiografias convencionais mostraram-se mais eficientes do que aquelas obtidas em imagens digitalizadas. Nimkarn e Miles ${ }^{54}$ concluíram ter havido uma ampliação horizontal na imagem digitalizada, ao compararem os valores das medidas assim obtidas, com aqueles obtidos pelo método manual. Os autores Tsang e Cooke $^{73}$ recomendaram cautela na utilização de 
medidas obtidas por meio de um programa de traçado cefalométrico; entretanto devendo-se salientar que o programa por eles utilizado, o Digigraph, demarca os pontos cefalométricos diretamente sobre a pele, tendo mostrado maior variabilidade nas medidas, quando comparado ao Dentofacial Planner. Outro fator importante a destacar, é que o programa Digigraph estima os pontos cefalométricos localizados em regiões esqueléticas, utilizando cálculos matemáticos. Tal estimativa poderia explicar a maior variabilidade encontrada nos valores das medidas. Em contrapartida, nas pesquisas de Bergin, Hallenberg, Malmgren"; Davis, Mackay ${ }^{20}$; Sandler ${ }^{66}$ e Oliver ${ }^{55}$, o método computadorizado mostrou melhor desempenho em relação ao método manual.

A escolha da metodologia adequada para avaliações cefalométricas comparativas em trabalhos desta natureza foi discutida na literatura por alguns autores. Houston ${ }^{34}$ e Sandler ${ }^{66}$ utilizaram em seus estudos o teste não paramétrico de Wilcoxon, justificando o fato dos valores encontrados não estarem distribuídos na curva de normalidade. Em 1983, Houston ${ }^{35}$ publicou um trabalho discorrendo a respeito da necessidade da realização da pesquisa de erros em medições cefalométricas, propondo os testes paramétricos, inclusive afirmando sua observação de que o teste $t$ de Student e a análise de variância predominaram como métodos eleitos para comparações intergrupos. Vários trabalhos consultados na literatura realizaram suas comparações por meio de testes paramétricos, entretanto não fizeram nenhuma observação quanto aos pré-requisitos necessários para a realização destes $^{5,9,13,16,18,20,25,37,49,50,54,55,58,73}$. Bland e Altman ${ }^{11}$ demonstraram que uma avaliação satisfatória de variação das medidas pode ser obtida examinando-se os limites de concordância ( \pm 2 S.D.). Afirmaram ainda que a forma estatística correta não é óbvia, propondo uma análise alternativa. Tal método estatístico foi empregado para comparar todas as medidas lineares e angulares no trabalho de Lowey $^{50}$.
Neste trabalho, provavelmente devido às características heterogêneas da amostra, no tocante às variadas faixas etárias e também ao fato desta provir de indivíduos de ambos os gêneros, apenas as medidas obtidas para as variáveis PoOr.GoGn, Co-Gn, S-Go, P-Nperp, $\overline{1}$-NB e diferença incluíram-se na curva de normalidade, possibilitando a comparação intergrupos por meio do teste paramétrico da análise de variância (ANOVA). Para as demais medidas, pelo fato de não preencherem os critérios de enquadramento na curva de normalidade, a comparação intergrupos foi realizada por meio do teste não paramétrico de Kruskall-Wallis, concordando com os trabalhos de Sandler ${ }^{66}$ e de Houston $^{34}$, que utilizaram o teste não paramétrico de Wilcoxon.

Ao analisar-se as vantagens e desvantagens da utilização dos diferentes métodos de traçado cefalométrico, pode-se inferir que o método de traçado manual, também denominado convencional, vem a ser um dos mais estudados em relação à confiabilidade de obtenção da localização dos pontos cefalométricos e de medições propriamente ditas. Muitas das pesquisas consultadas enfocaram aspectos inter e intra-examinadores, realizando comparações entre métodos de traçado cefalométricos distintos ${ }^{1-7,10,13,18,27,29,31,34,37,45,46,49,51-53,59,65-67,69-}$ 71,77. Algumas delas correlacionaram a reprodutibilidade das medições diversas à experiência do operador $^{3,27,46,69}$.

Além disto, vem a constituir o método menos oneroso, pelo pouco material necessário no seu emprego. A grande desvantagem recai sobre o fato de que, por necessitar de um tempo maior de execução, tarefas muito extensas, onde muitas radiografias estão envolvidas e um grande número de medições são requeridas, podem tornar-se tediosas e desgastantes para o operador, ao contrário do método computadorizado $4,5,9,12,17,33,34,35,44,58$.

A digitalização das imagens dos traçados foi uma adaptação realizada, com o objetivo de equipará-la ao uso do programa Dentofacial Planner 7.02. Também constituiu-se na forma de verifi- 
cação de possíveis distorções ocorridas durante a etapa de digitalização destas imagens. Na literatura, foi possivel perceber uma abordagem análoga neste sentido, apenas no trabalho de Krogstad e $\mathrm{Kvam}^{45}$, realizado em 1971, fundamentado em erros geométricos nas medições, influenciados por ampliações entre os lados direito e esquerdo. Por meio dos resultados obtidos, os autores concluíram ter havido distorção da imagem, pelo fato de algumas medidas, principalmente aquelas obtidas mais à direita do cefalograma, terem se apresentado sempre aumentadas. A digitalização de traçados também foi empregada comparativamente nos trabalhos de Cohen ${ }^{18}$; Liu, Gravely ${ }^{49}$ e Sandler ${ }^{66}$, entretanto, não houve nenhuma referência a possíveis ocorrências de distorção na imagem.

Pelos resultados obtidos, onde não se verificou a ocorrência de distorções das imagens, ficou evidente a possibilidade de utilização do programa também desta forma adaptada. Entretanto, assim procedendo, perder-se-ia um pouco da rapidez nas medições, em vez de utilizá-lo da forma preconizada pelo fabricante.

A digitalização das radiografias, de forma a ser utilizada para medições cefalométricas, tornou-se o método preferencialmente escolhido, nos dias de hoje. À medida que a tecnologia progride, oferece facilitações para adequar o homem às muitas tarefas diárias da rotina profissional, e necessita fazer isto com garantia de precisão. No campo da cefalometria radiográfica, este trabalho de pesquisa, assim como outros $5,13,16,20,37,50,54,55,58,66$, vem comprovar a eficiência alcançada pelos recursos tecnológicos, principalmente no âmbito de pesquisa. Apenas no trabalho de Forsyth et al. ${ }^{25}$, realizado em 1996, a imagem digital teve um desempenho pior do que a da radiografia convencional, em pontos localizados em margens indefinidas, como o N e o A. Apesar desta comprovação, convém ressaltar que a qualidade do equipamento utilizado influencia diretamente nos resultados, em contrapartida elevando os custos, conforme salientado por Lowey ${ }^{50}$; Liu e Gravely ${ }^{49}$. Além da qualidade do equipamento em si, torna-se conveniente lembrar que os programas de traçado cefalométrico mais modernos disponibilizam ferramentas que permitem o delineamento provisório de determinadas estruturas sobre as quais determinadas marcações de pontos eram mais difíceis, como citaram Forsyth et al. ${ }^{25}$. Adequado e comercializado principalmente para uso clínico, as análises fornecidas pelo Radiocef 2.0 não preenchiam a necessidade das medições a serem realizadas nesta pesquisa. Porém, o programa forneceu as ferramentas necessárias a esta adequação, tendo sido elaborada uma análise própria para as medições selecionadas. Nesta etapa de elaboração, houve a preocupação de garantir que as medições lineares estivessem sendo obtidas dentro das categorias disponibilizadas pelo programa Dentofacial Planner 7.02; ou seja, medidas de comprimento, distâncias horizontais e verticais e distâncias ponto-linha. Tal questão foi resolvida de forma relativamente simples.

A principal dificuldade quando da utilização deste método foi observada durante a localização dos pontos nos ápices dentários dos incisivos, onde a imagem digitalizada oferece tons de cinza que se confundem nesta região. Mesmo com a utilização dos recursos disponibilizados pelo programa, como a filtragem ou o zoom, esta localização ainda é mais difícil em relação às películas radiográficas. Entretanto, pôde-se perceber ser este fato comum a outros métodos e mesmo a outros programas incluídos nesta categoria ${ }^{6,13,27,49,66,69}$. Ainda, à medida que se vai familiarizando com a imagem, o grau de dificuldade de localização dos pontos nestes locais, assim como em outros locais, de forma geral, tende a diminuir.

Uma maneira apropriada de melhorar a qualidade da imagem digitalizada implica na utilização de um monitor de vídeo de alta resolução. Isto influencia sobremaneira na visualização de detalhes da imagem. A forma de captura da imagem também precisa ser adequadamente realizada, segundo as instruções do fabricante.

Com vistas à utilização do programa para fins 
de pesquisa, este necessitava ainda permitir exportar os dados e imagens para outros programas. Neste estudo, todos os dados tiveram que ser digitados manualmente no programa estatístico, que além de consumir um tempo muito grande, ficam sujeitos a erros. A versão 2000 do Radiocef já sofreu melhorias, possibilitando a exportação das imagens; entretanto ainda não permitindo a exportação dos dados obtidos das medidas.

O programa Dentofacial Planner, por ter sido lançado há mais tempo, e em outro país, já foi objeto de outras pesquisas, onde de uma maneira geral mostrou-se eficiente ${ }^{3,5,21,24,39,40,41,48,51,56,76}$. Ele pode ser utilizado de duas maneiras: digitalizando-se os pontos sobre o traçado realizado manualmente, ou diretamente sobre as radiografias, ambas na mesa digitalizadora. A nova versão do programa, associada ao programa Showcase 2.1, possibilita trabalhar com imagens radiográficas digitalizadas.

A sua utilização eficientemente comprovada constituiu-se a razão de ter sido eleito como parâmetro de comparação em relação ao programa nacional Radiocef 2.0. Este, conforme explicado, foi alvo inclusive de uma adaptação com vistas a operar o mais semelhantemente possivel em relação ao Dentofacial Planner 7.02, desta forma permitindo avaliar e localizar possíveis distorções quando da digitalização das imagens.

\section{CONCLUSÕES}

De acordo com a metodologia empregada, e a partir dos resultados obtidos na comparação entre o programa de traçado cefalométrico Radiocef 2.0, o método manual e o programa Dentofacial Planner 7.02, pôde-se concluir que:

1) o programa de traçado cefalométrico computadorizado Radiocef 2.0 pode ser confiavelmente utilizado como recurso auxiliar de diagnóstico, plano de tratamento, acompanhamento e avaliação de tratamentos ortodônticos, nos âmbitos clínico e/ou de pesquisa;

2) este programa também pode ser confiavelmente utilizado para efetuar medições a partir da digitalização dos traçados, além da forma proposta pelo fabricante.

\title{
Evaluation of a cephalometric software
}

\begin{abstract}
Aim: to evaluate the cephalometric software Radiocef 2.0 regarding reliability and precision, as compared to the conventional manual tracing and measuring procedures, and to the software Dentofacial Planner 7.02 (Gold Standard). Methods: the sample consisted of 50 good quality lateral head films from patients of both gender at the 11 to 24-year age range belonging to the files of the orthodontic graduate clinic at Bauru Dental School, University of São Paulo. The radiographs were traced and measured by different methodologies generating four groups as follows: Group 1 (the radiographs were manually traced and measured); Group 2 (the cephalograms and landmarks were scanned and measured with Radiocef 2.0 software); Group 3 (the radiographs were scanned and measured with Radiocef 2.0 software); and Group 4 (the landmarks were digitized and measured with Dentofacial Planner 7.02 software). Linear and angular measurements were performed and an analysis of variance and a Kruskal-Wallis test were conducted for intergroup comparison. Results: results demonstrated no statistically significant differences between the groups. Conclusions: the software is reliable to be used for clinical and investigative purposes and that Radiocef 2.0 can be used by either scanning the cephalograms and landmarks or by scanning the radiograph itself.
\end{abstract}

Key words: Computerized cephalometrics. Computerized cephalometric tracing. Cephalometric software. 


\section{REFERÊNCIAS}

1. ADAMS, J. W. Correction of error in cephalometric roentgenograms. Angle Orthod, Appleton, v. 10, no. 1, p. 3-13, Jan. 1940.

2. ADENWALLA, S. T.; KRONMAN, J. H.; ATTARZADEH, F. Porion and condyle as cephalometric landmarks: an error study. Am J Orthod Dentofacial Orthop, St. Louis, v. 94, no. 5, p. 411-5, Nov. 1988.

3. AlBUQUERQUE JR., H. R.; ALMEIDA, M. H. C. Avaliação do erro de reprodutibilidade dos valores cefalométricos aplicados na filosofia Tweed-Merrifield, pelos métodos computadorizado e convencional. Ortodontia, São Paulo, v. 31, n. 3, p.18-30, set./dez. 1998.

4. BARRETT, M. J.; BROWN, T.; McNULTY, E. C. A computer-based system of dental and cranio-facial measurement and analysis. Aust Dent J, Sydney, v.13, no. 3, p. 207-12, June 1968.

5. BASKIN, N. H.; CISNEROS, G. J. A comparison of two computer cephalometric programs. J Clin Orthod, Boulder, v. 31, no. 4, p. 231-3, Apr. 1997.

6. BAUMRIMD, S.; FRANTZ, R. C. The reability of head film measurements; 1: landmark identification. Am J Orthod, St. Louis, v. 60 , no. 2, p.111-27, Aug. 1971

7. BAUMRIND, S.; FRANTZ, R. C. The reability of film measurements; 2: conventional angular and linear measures. Am J Orthod, St. Louis, v. 60, no. 5, p. 505-17, Nov. 1971.

8. BAUMRIND, S.; MILLER, D. M. Computer-aided head film analysis: the University of California San Francisco method. Am J Orthod, St. Louis, v. 78, no. 1, p. 41-65, Jul. 1980.

9. BERGIN, R.; HALLENBERG, J.; MALMGREN, O. Computerized cephalometrics. Acta Odont Scand, Oslo, v. 36, p. 349-57, Jul. 1978.

10. BJÖRK, A.; SOLOW, B. Measurement on radiographs. J Dent Res, Chicago, v. 41, no. 3, p. 672-83, May/June, 1962.

11. BLAND, J. M.; ALTMAN, D. G. Statistical methods for assessing agreement between two methods of clinical measurement. Lancet, London, v. 1, p. 307-10, 1986.

12. BONDEVIK, O.: ROSLER, M.: SLAGSVOLD, O. The digital readout system $\mathrm{CM}-1$; an instrument for rational measuring on radiographic headplates and dental models. Eur J Orthod, London, v. 3, no. 1, p. 1-8, 1981.

13. BRANGELI, L. A. M. et al. Estudo comparativo da análise cefalométrica pelo método manual e computadorizado. Rev Ass Paul Cirurg Dent, São Paulo, v. 54, n. 3, p. 234-41, maio/jun. 2000.

14. BROADBENT, B. H. A new x-ray technique and its application to Orthodontics. Angle Orthod, Appleton, v. 1, no. 2, p. 45-66, Apr. 1931

15. CDT - Consultoria, Desenvolvimento, Treinamento em Informática. Disponível em: <http://www.cdt.com.br>. Acesso em: 20 abr. 2000.

16. CHACONAS, S. J.; JACOBSON, R. L.; LEMCHEN, M. S. The digigraph work station; part 3: accuracy of cephalometric analysis. J Clin Orthod, Boulder, v. 24, no. 8, p. 467-71, Aug. 1990.

17. CHEBIB, F. S.; CLEALL, J. F.; CARPENTER, K. J. On-line computer system for the analysis of cephalometric radiographs. Angle Orthod, Appleton, v. 46, no. 4, p. 305-11, Oct. 1976.

18. COHEN, A. M. Uncertainty in cephalometrics. Br J Orthod, London, v. 11, no. 1, p. 44-8, Jan. 1984.

19. DAHLBERG, G. Statistical methods for medical and biological students. New York: Interscience, 1940

20. DAVIS, D. N.; MACKAY, F. Reability of cephalometric analysis using manual and interactive computer methods. Br J Orthod, London, v. 18, no. 2, p. 105-9, May, 1991.

21. DEBERARDINIS, M. et al. Evaluation of the vertical holding appliance in treatment of high-angle patients. Am J Orthod Dentofacial Orthop, St. Louis, v. 117, no. 6, p. 700-5, June, 2000.

22. DENTOFACIAL SOFTWARE INC. Dentofacial planner user manual: Version 7.02. Toronto. [s.n., 19]

23. FABER, R. D.; BURSTONE, C. B.; SOLONCHE, D. J. Computerized interactive orthodontic treatment planning. Am J Orthod, St. Louis, v. 73, no. 1, p. 36-46, Jan. 1978.
24. FISCHER-BRANDIES, H. et al. Predictability using the "Dentofacial Planner version 4.22 A: soft tissue changes following adult orthodontic treatment. Inf Orthod Kieferorthop, München, v. 22 , no. 4 , p. $517-24,1990$

25. FORSYTH, D. B. et al. Digital imaging of cephalometric radiographs, part 2: image quality. Angle Orthod, Appleton, v. 66, no. 1, p. 43-50, 1996

26. FRANKLIN, J. B.; MILWAUKEE, W. Certain factors of aberration to be considered in clinical roentgenographic cephalometry Am J Orthod, St. Louis, v. 38, no. 5, p. 351-68, 1952.

27. GRAVELY, J. F.; BENZIES, P. M. The clinical significance of tracing error in cephalometry. Br J Orthod, London, v. 1, no. 3, p. 95-101, 1974

28. HALAZONETIS, D. J. Computer-assisted cephalometric analysis. Am J Orthod Dentofacial Orthop, St. Louis, v.105, no. 5, p. 517-21, May 1994

29. HATTON, M. E.; GRAINGER, R. M. Reability of measurements from cephalograms at the Burlington orthodontic research centre. J Dent Res, Chicago, v. 37, no. 5, Sept./Oct. 1958.

30. HING, N. R. The accuracy of computer generated prediction tracings. Int J Oral Maxillofac Surg, Copenhagen, v. 18, no. 3, p.148-51, June, 1989.

31. HIXON, E. H. The norm concept and cephalometrics. Am J Orthod, St. Louis, v. 42, no. 12, p. 898-906, Dec. 1956.

32. HOFRATH, H. Die bedeutung der Röntgenfern und Abstandsaufnahme für die Diagnostik der Kieferanomalien. Fortschr Orthodont, München, v. 1, p. 232-58, Apr./Jul, 1931.

33. HOUSTON, W. J. B. The application of computer aided digital analysis to orthodontic records. Eur J Orthod, London, v. 1 no. 2, p. 71-9, 1979.

34. HOUSTON, W. J. B. A comparison of the reability of measurements of cephalometric radiographs by tracings and direct digitization. Swed Dent J Suppement, Malmö, v.15, p. 99-103, 1982.

35. HOUSTON, W. J. B. The analysis of errors in orthodontic measurements. Am J Orthod, St. Louis, v. 83, no.5, p. 382-90, May, 1983.

36. Image Scan International Ltd. Suite 10. Raglan House: Llantarnam Park, Cwmbran, gwent NP4 3AX.

37. JACKSON, P. H.; DICKSON, G. C.: BIRNIE, D. J. Digital image processing of cephalometric radiographs: a preliminary report. Br J Orthod, London, v. 12, p. 122-32, Jul. 1985.

38. JANDEL Corp. Disponível em: <http://www.spss.com/sigmastat>. Acesso em: 20 abr. 2005

39. JANSON, G. R. P. Estudo tridimensional das assimetrias dentárias e esqueléticas na má oclusão de Classe II, subdivisão. 1998. 271f. Tese (Livre Docência)-Faculdade de Odontologia de Bauru, Universidade de São Paulo, Bauru, 1998.

40. JANSON, G. R. P.; METAXAS, A.; WOODSIDE, D. G. Variation in maxillary and mandibular molar and incisor vertical dimension in 12-year-old subjects with excess, normal, and short lower anterior face height. Am J Orthod Dentofacial Orthop, St. Louis, v. 106, no. 4, p. 409-18, Oct. 1994.

41. JANSON, G. R. P. et al. Eruption guidance appliance effects in the treatment of Class II, division 1 malocclusions. Am J Orthod Dentofacial Orthop, St. Louis, v. 117, no. 2, p. 119-29, Feb. 2000

42. KONCHAK, P. A.; KOEHLER, J. A. A Pascal computer program for digitizing lateral cephalometric radiographs. Am J Orthod St. Louis, v. 87, no. 3, p. 197-200, Mar. 1985.

43. KROGMAN, W. M. Validation of the roentgenographic cephalometric technique. Am J Orthod, St. Louis, v. 44, no. 12, p. 933-9, Dec. 1958.

44. KROGMAN, W. Use of computer in orthodontic analysis and diagnosis: a symposium. Am J Orthod, St. Louis, v. 61, no. 3 p. 219-20, Mar. 1972

45. KROGSTAD, O.; KVAM, E. Geometric errors in measurements on x-rays films. A methodologic study on lateral model exposures. Acta Odont Scand, Oslo, v. 29, no. 2, p. 185-96, Jun 1971.

46. KVAM, E.; KROGSTAD, O. Variability in tracings of lateral head plates for diagnostic orthodontic purposes: a methodologic study. Acta Odont Scand, Oslo, v. 27, no. 4, p. 359-69, Aug. 1969. 
47. LANSOFT Informatical. Disponível em: <http://www.geocities.com/Hotsprings. 20 abr. 2000

48. LEITAO, P.; NANDA, R. S. Relationship of natural head position to craniofacial morphology. Am J Orthod Dentofacial Orthop St. Louis, v. 117, no. 4, p. 406-17, Apr. 2000.

49. LIU, Y; GRAVELY, J. F. The reability of the "Ortho Grid" in cephalometric assessment. Br J Orthod, London v. 18, p. 21-7, Feb. 1991.

50. LOWEY, M. N. The development of a new method of cephalometric and study cast mensuration with a computer controlled video image capture system; part I: video image capture system. Br J Orthod, London, v. 20, no. 3, p. 203-14, 1993.

51. MARTINS, L. P. et al. Erro de reprodutibilidade das medidas cefalométricas das análises de Steiner e de Ricketts, pelo método convencional e pelo método computadorizado. Ortodontia, São Paulo, v. 28, n. 1, p. 4-17, jan./abr. 1995.

52. MCWILLIAM, J. S.; WELANDER, U. The effect of image quality on the identification of cephalometric landmarks. Angle Orthod, Appleton, v. 48, no. 1, p. 49-56, Jan. 1978.

53. MIDTGARD, J.; BJÖRK, G.; LINDER-ARONSON, S. Reproducibility of cephalometric landmarks and errors of measurements of cephalometric cranial distances. Angle Orthod, Appleton, v. 44. no.1, p. 56-61, Jan. 1974.

54. NIMKARN, Y.; MILES, P. G. Reliability of computer-generated cephalometrics. Int J Adult Orthodon Orthognath Surg, Chicago, v. 10 , no. 1 , p. $43-52,1995$

55. OLIVER, R. G. Cephalometric analysis comparing five different methods. Br J Orthod, London, v. 18, no. 4, p. 277-83, Nov. 1991.

56. RABIE, A. B. M.; GU, Y. Diagnostic criteria for pseudo-Class III malocclusion. Am J Orthod Dentofacial Orthop, St. Louis, v. 117, no. 1, p. 1-9, Jan. 2000.

57. RADIOMEMORY. Manual do programa Radiocef 2.0. Belo Horizonte: Ed. Brasil, 1998.

58. RICHARDSON, A. A comparison of tradicional and computerized methods of cephalometric analysis. Eur J Orthod, London, v. 3, p. 15-20, 1981.

59. RICKETTS, R. M. A foundation for cephalometric communication. Am J Orthod, St. Louis, v. 46, no. 5, p. 330-57, May, 1960.

60. RICKETTS, R. M. The evolution of diagnosis to computerized cephalometrics. Am J Orthod, St. Louis, v. 55, no. 6, p. 795-803, June 1969.

61. RICKETTS, R. M. et al. An overview of computerized cephalometrics. Am J Orthod, St. Louis, v. 61, no. 1, p. 1-28, Jan. 1972.
62. RICKETTS, R. M. Perspectives in the clinical application of cephalometrics; the first fifty years. Angle Orthod, Appleton v. 51, no. 2, p. 115-50, Apr. 1981

63. ROCKY MOUNTAIN DATA SYSTEMS. Computerized cephalometrics manual. Revised edition by Sherman Oaks. California: RDMS, 1973.

64. RUDOLPH, D. J.; SINCLAIR, P. M. COGGINS, J. M. Automatic computerized radiographic identification of cephalometric landmarks. Am J Orthod Dentofacial Orthop, St. Louis, v. 113, no. 2, p. 173-9, Feb. 1998.

65. SALZMANN, J. A. Limitations of roentgenographic cephalometrics. Am J Orthod, St. Louis, v. 50, no. 3, p. 169-88, Mar. 1964.

66. SANDLER, P. J. Reproducibility of cephalometric measurements. Br J Orthod, London, v. 15, no. 2, p. 105-10, May 1988.

67. SEKIGUCHI, T.; SAVARA, B. S. Variability of cephalometric landmarks used for face growth studies Am J Orthod, St. Louis, v. 61, no. 6, p. 603-18, June, 1972.

68. SOFT Manager. Disponível em: <http://www.softmanager. com.br>. Acesso em: 20 abr. 2000.

69. STABRUN, A. E.; DANIELSEN, K. Precision in cephalometric landmark identification. Eur J Orthod, London, v. 4, p. 185-96, 1982.

70. STEINER, C. C. Cephalometrics for you and me. Am J Orthod St. Louis, v. 39, no. 10, p. 729-55, Oct. 1953

71. STEINER, C. C. The use of cephalometrics as an aid to planning and assessing orthodontic treatment. Am J Orthod, St. Louis, v. 46, no.10, p. 721-35, Oct. 1960

72. TOIGO, E.; MORO, A.; VIGORITO, J. W. Proposta de um programa computadorizado para a análise de Petrovic/ Lavergne. Ortodontia, São Paulo, v. 29, no. 2, p. 16-26, maio/ago. 1996.

73. TSANG, K. H.; COOKE, M. S. Comparison of cephalometric analysis using a non-radiographic sonic digitizer (Digigraph Workstation) with conventional radiography. Eur J Orthod, London, v. 21 , no. 1 , p. 1-13, 1999

74. TWEED, C. W. Why I extract teeth in the treatment of certain types of malocclusion. Alpha Omegan, New York, v. 46 p. 93-104, 1952

75. WALKER, R. P. Dentofacial Planner ${ }^{\mathrm{TM}}$ : diagnosis and treatment planning software for orthodontics and orthognathic surgery. [s.l., s.n.], 1990.

76. WEST, K. S.; McNAMARA, J. A. Changes in the craniofacial complex from adolescence to midadulthood, a cephalometric study. Am J Orthod Dentofacial Orthop, St. Louis, v. 115 no. 5, p. 521-32, May 1999.

77. YEN, P. K. J. Identification of landmarks in cephalometric radiographs. Angle Orthod, Appleton, v. 30, no. 1, p. 35, 41, Jan. 1960.

\section{Endereço para correspondência}

Guilherme Janson

Universidade de São Paulo - Faculdade de Odontologia de Bauru Departamento de Odontopediatria, Ortodontia e Saúde Coletiva Alameda - Dr. Octávio Pinheiro Brisolla, 9-75 - Vila Universitária CEP: 17.043-101 - Bauru/SP

E-mail: jansong@travelnet.com.br 